CASE REPORT

\title{
Retrieval of a firmly impacted knotted coronary catheter through the contralateral femoral artery using a balloon angioplasty catheter
}

Syed Hasnain Mujtaba MBBS MRCP ${ }^{1}$, Finn Farquharson MBBS MSc FRCR ${ }^{2}$, Samer Arnous MBBS MRCPI ${ }^{1}$, Magdi El-Omar BSc MBBS MRCP MD ${ }^{1}$

SH Mujtaba, F Farquharson, S Arnous, M El-Omar. Retrieval of a firmly impacted knotted coronary catheter through the contralateral femoral artery using a balloon angioplasty catheter. Curr Res Cardiol 2014;1(2):107-110.

Knotting of a coronary catheter during cardiac catheterization is a relatively common event and can usually be resolved by simply untwisting the catheter or by straightening it with a guidewire. If these manoeuvres fail, the knotted catheter may be retrieved using a snare device; however, if unsuccessful, surgical extraction may prove necessary. The authors describe a case in which a snare device failed to retrieve a knotted catheter firmly impacted within a loop in the external iliac artery. The catheter was successfully mobilized using an angioplasty balloon inflated distal to the site of impaction then pulled back, allowing dislodgement of the knot and subsequent extraction of the catheter from the contralateral femoral artery.

Key Words: Angioplasty; Balloon; Catheter; Knotted; Snare

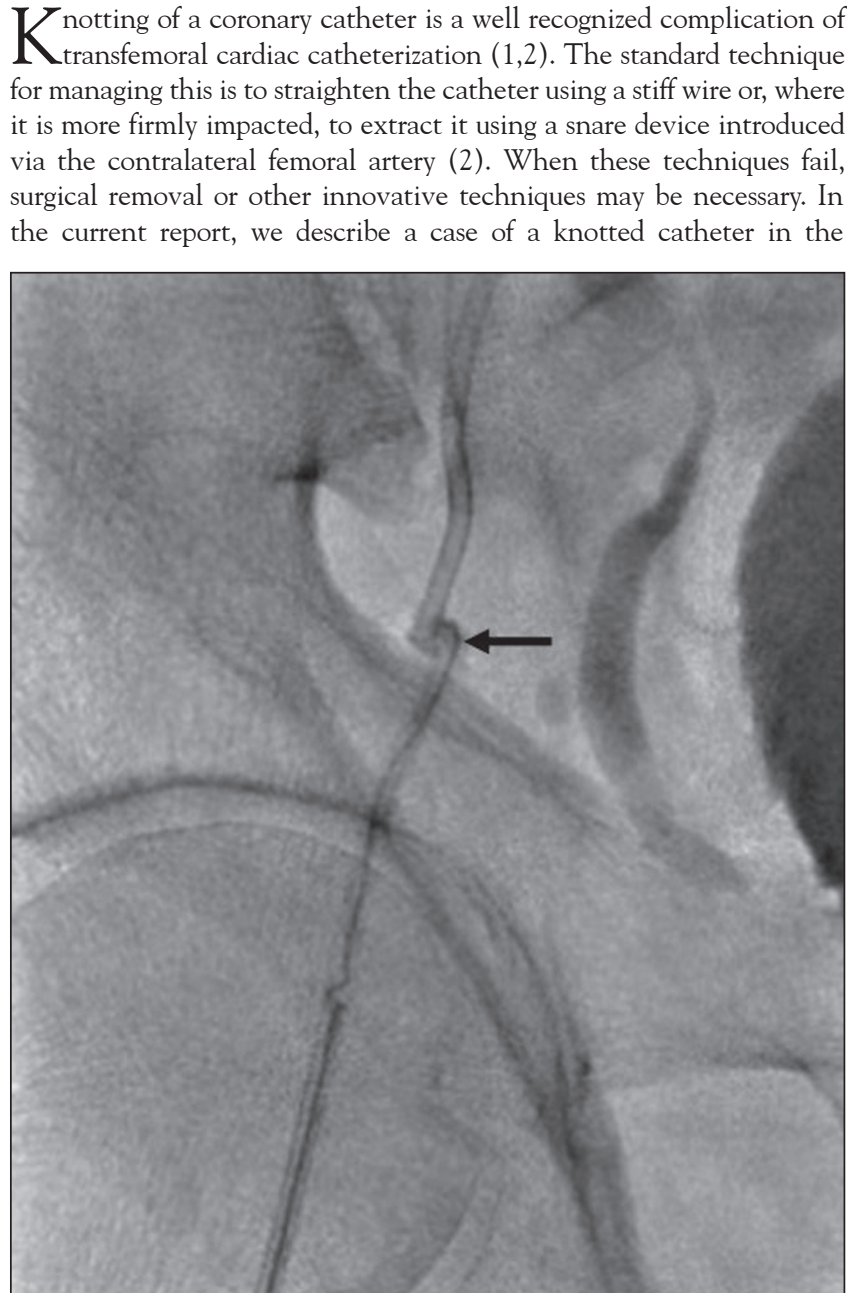

Figure 1) Distal tip of the guidewire (Terumo Medical Corporation, USA) entrapped within the catheter knot (arrow)

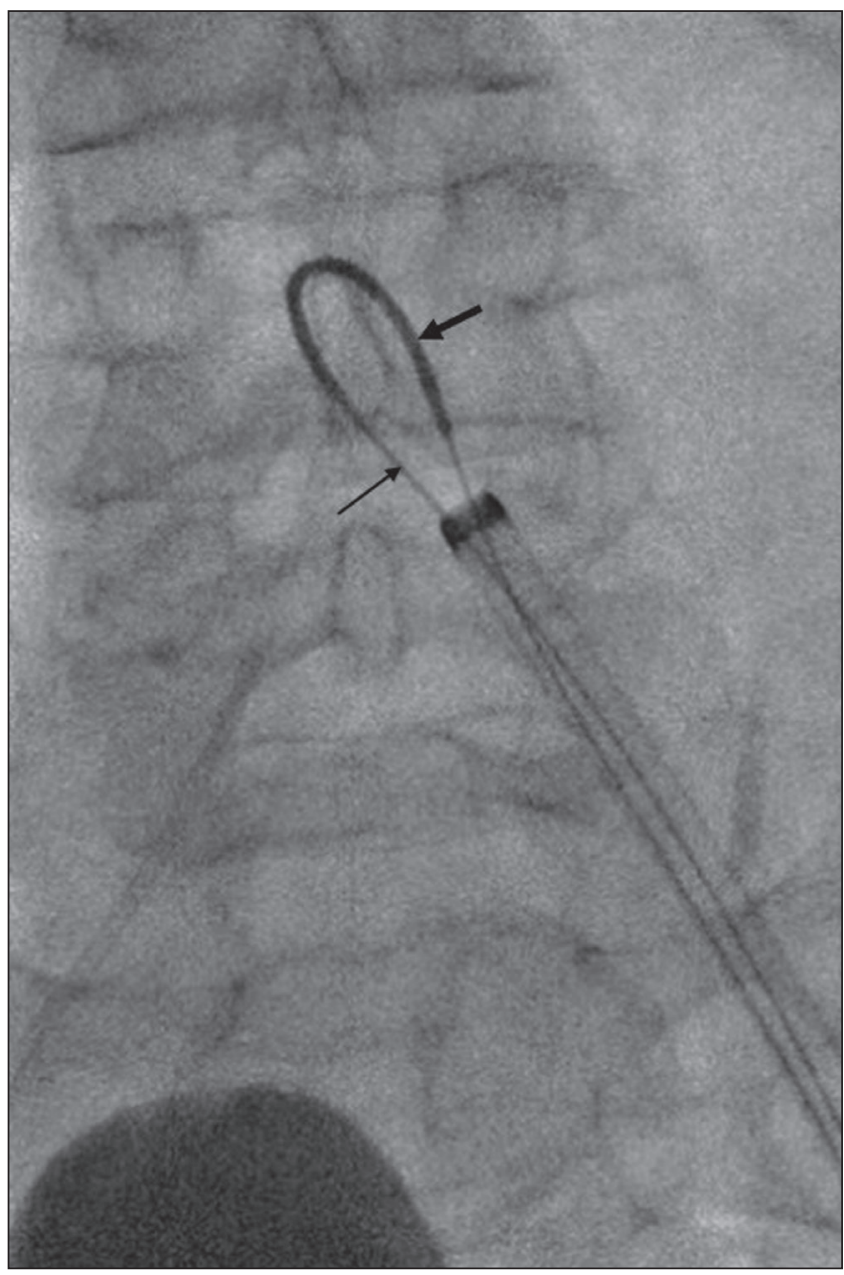

Figure 2) Guidewire (Terumo Medical Corporation, USA) (small arrow) within SOS Omni (Angiodynamics, USA) catheter (large arrow) snaring around trapped catheter

\footnotetext{
${ }^{1}$ Department of Cardiology, Manchester Heart Centre, Manchester Royal Infirmary; ${ }^{2}$ Department of Radiology, Manchester Royal Infirmary, Manchester, United Kingdom

Correspondence: Dr Magdi El-Omar, Department of Cardiology, Manchester Heart Centre, Manchester Royal Infirmary, Oxford Road, Manchester, M13 9WL United Kingdom. Telephone 44-1612761234, fax 44-1612767956, e-mail magdi.el-omar@cmft.nhs.uk
} 


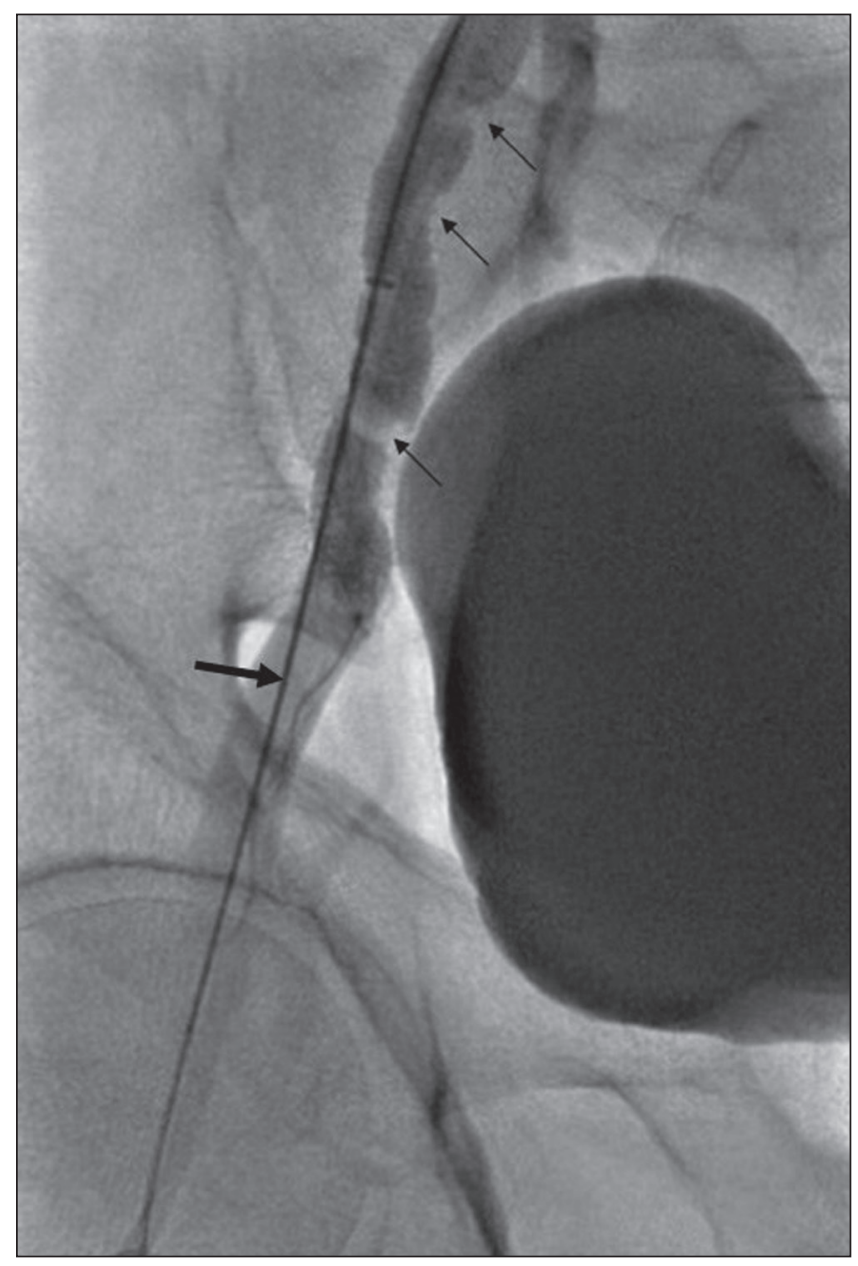

Figure 3) 'Accordion' effect (small arrows) apparent within the external iliac artery as a result of straightening with a super-stiff guidewire (large arrow)

external iliac artery (EIA) that could not be extracted in its entirety using standard techniques, including a snare catheter. The knotted part of the catheter was successfully mobilized using an angioplasty balloon inflated just distal to the site of impaction, then pulled back toward the contralateral femoral artery, enabling its subsequent extraction.

\section{CASE PRESENTATION}

A 68-year-old man underwent coronary angiography following presentation with a non-ST elevation myocardial infarction. Arterial access was obtained via the right common femoral artery (CFA) using a short $(11 \mathrm{~cm}) 6 \mathrm{Fr}$ femoral sheath (Cordis Avanti+, Cordis Corporation, USA). Left coronary angiography, using a 6 Fr JL4 diagnostic coronary catheter (Cordis Super Torque Plus $100 \mathrm{~cm} / 2.0 \mathrm{~mm}$ ), revealed moderate atheroma in the proximal left anterior descending artery and a normal left circumflex artery. Following exchange over a standard 0.035-inch J-tipped guide wire (Cordis Emerald $150 \mathrm{~cm}$ ), a 6 Fr JR4 diagnostic coronary catheter (Cordis Super Torque Plus $100 \mathrm{~cm} / 2.0 \mathrm{~mm}$ ) was used for right coronary artery (RCA) angiography, revealing moderate disease in its proximal segment. A pressure wire study to the left anterior descending artery was pursued using a $6 \mathrm{Fr} J L 4$ guide catheter. The fractional flow reserve was measured at 0.91 post-adenosine infusion, indicating non-flow limiting disease. The intention was then to proceed to a pressure wire study to the proximal RCA. The previous catheter was exchanged for a 6 Fr JR4 guide catheter over a 0.035 -inch guide wire. In the process of attempting to engage the RCA ostium, torquing the catheter became increasingly difficult and catheter knotting was suspected. An attempt was made to remove the catheter at

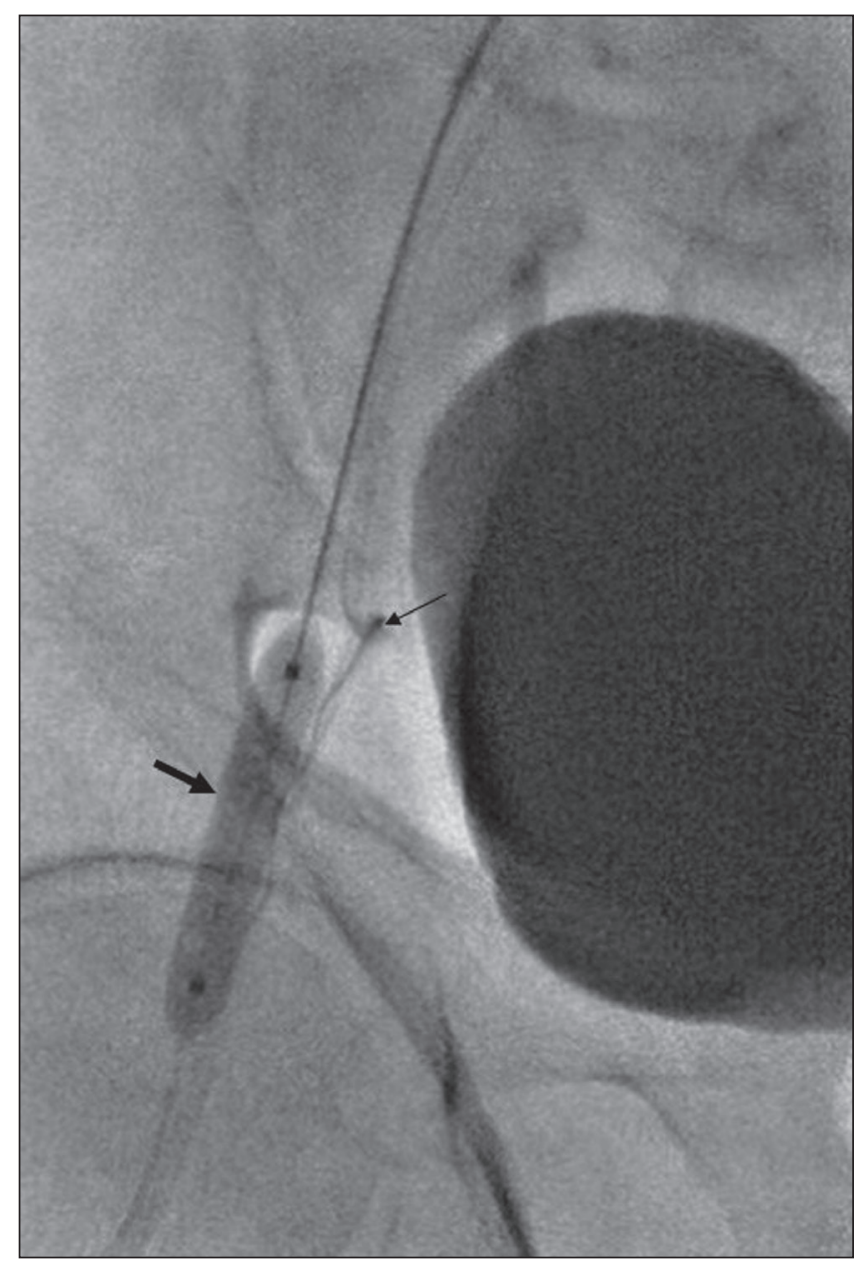

Figure 4) Angioplasty balloon (large arrow) inflated just distal to the catheter knot (small arrow)

this stage, but only a short portion could be exteriorized before resistance was encountered. Fluoroscopy of the right groin revealed a kink followed by a knot within the catheter segment in the right EIA. Attempts at untwisting the catheter using counter-torque proved unsuccessful. It was noted at this stage that the knotted catheter had become 'impacted' in the EIA, such that it was now impossible to move it in or out. An attempt was made to straighten the catheter using the J-tipped end, followed by the stiff end of a standard 0.035inch guide wire but this was unsuccessful. A similar attempt using an Amplatz Super-Stiff 0.035-inch wire (Boston Scientific, USA) also failed. An attempt was then made to advance an Asahi Sion angioplasty guidewire (Abbott Vascular, USA) $(0.014 \mathrm{inch} / 180 \mathrm{~cm}$ ) past the deformed segment of the catheter, but although this travelled beyond the proximal kink, it failed to cross the knot within the catheter. Finally, an attempt was made to advance a 0.035 -inch Terumo guidewire (Terumo Medical Systems, USA) past the kink, but its distal end became entrapped within the catheter knot (Figure 1) and attempts at removing it resulted in its snapping, with the free distal segment becoming entrapped within the knot. A further attempt at untwisting the catheter resulted in its snapping just outside the $6 \mathrm{Fr}$ sheath, presumably due to loss of the 'protective' effect of the sheath on catheter overtorquing at that point. An attempt was made at retrieval of the catheter through the contralateral femoral artery using a snare device. A 5 Fr sheath (Cordis Avanti+ $[11 \mathrm{~cm}]$ ) was inserted into the left CFA to enable placement of a $10 \mathrm{Fr}$ Prostar XL vascular closure device (Abbott Vascular, USA) for later hemostasis. A $16 \mathrm{Fr}$ sheath $(68.6 \mathrm{~cm}$; Cook Medical, USA) was then inserted into the left 


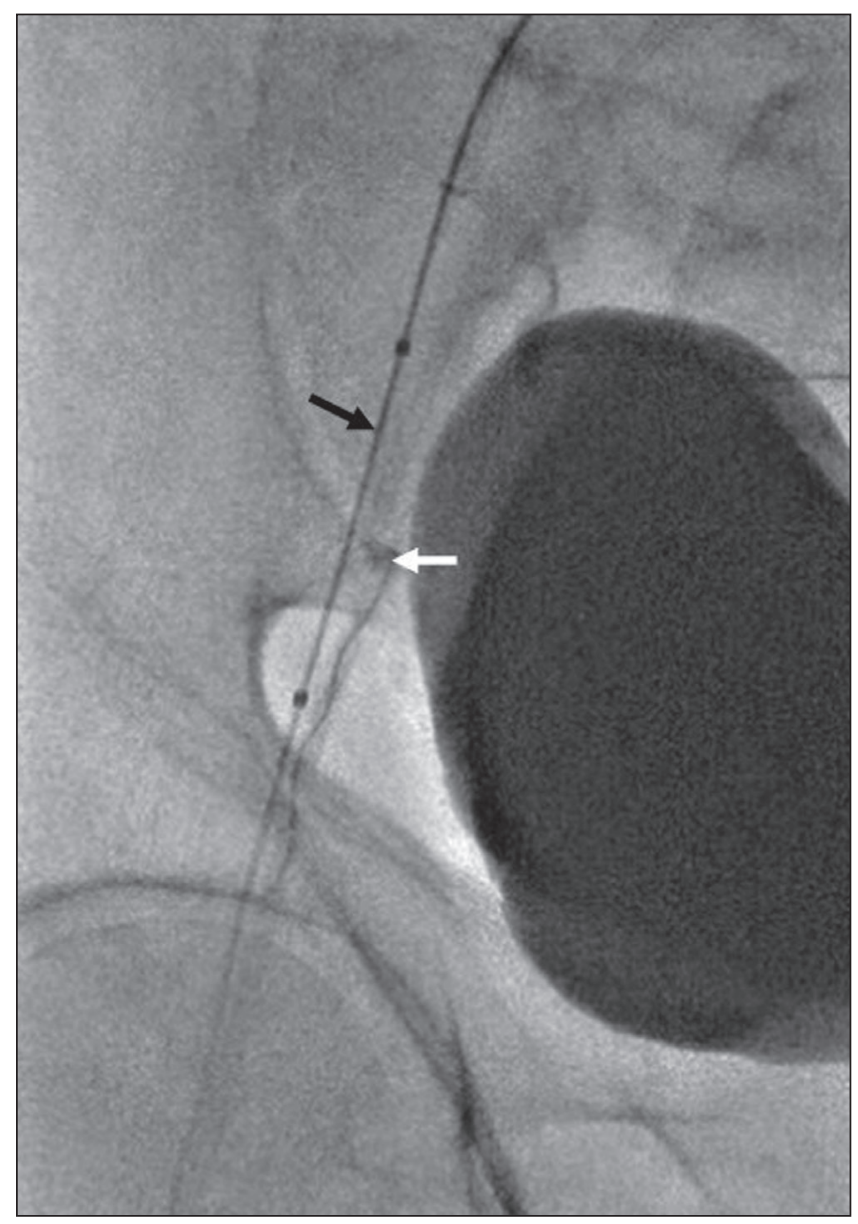

Figure 5) The angioplasty balloon, now deflated (black arrow), has been withdrawn to a more proximal position, dragging the catheter knot with it (white arrow) toward the aortic bifurcation (a 'toothpaste' effect)

CFA, and a 5 Fr SOS Omni selective catheter (Angiodynamics, USA) passed through it. A 6 Fr Amplatz GooseNeck $(15 \mathrm{~mm})$ snare catheter (Covidien, USA) was then passed alongside this. An exchange-length Terumo guidewire was inserted into the SOS Omni catheter that hooked over the knotted catheter. The snare catheter captured the Terumo guidewire and both the SOS Omni and snare catheters were then retrieved along with the distal part of the knotted guide catheter (Figure 2). The proximal end of the guide catheter, however, remained entrapped within the right EIA. With continued traction, the guide catheter snapped again just as it emerged from the 16 Fr sheath, leaving an intravascular catheter segment straddling the aortic bifurcation, with its ends protruding from the two groin sheaths.

A 7 Fr Terumo Pinnacle destination sheath $(2.5 \mathrm{~mm} / 45 \mathrm{~cm})$ was then inserted through the $16 \mathrm{Fr}$ sheath and advanced across the aortic bifurcation toward the right common iliac artery (CIA). An Amplatz Super-Stiff wire was passed through this and advanced well below the puncture site in the right CFA. An $8 \mathrm{~mm} \times 4 \mathrm{~cm}$ Cordis balloon was then passed over this wire and positioned just below the knot in the EIA. Fluoroscopy with contrast injection showed multiple invaginations (ie, 'accordion effect') within the EIA (Figure 3) due to multiple instrumentation and superimposed spasm. The balloon was then fully inflated manually using a $20 \mathrm{~mL}$ luer lock syringe containing a 50/50 contrast/saline mixture (Figure 4). The inflated balloon was pulled back gently to engage the knot, which lead to dislodgement of the knotted catheter remnant with a 'tooth pasting' effect (Figure 5). Under fluoroscopic guidance, the catheter remnant was then pulled out through the $16 \mathrm{Fr}$ left femoral artery sheath. Further fluoroscopy

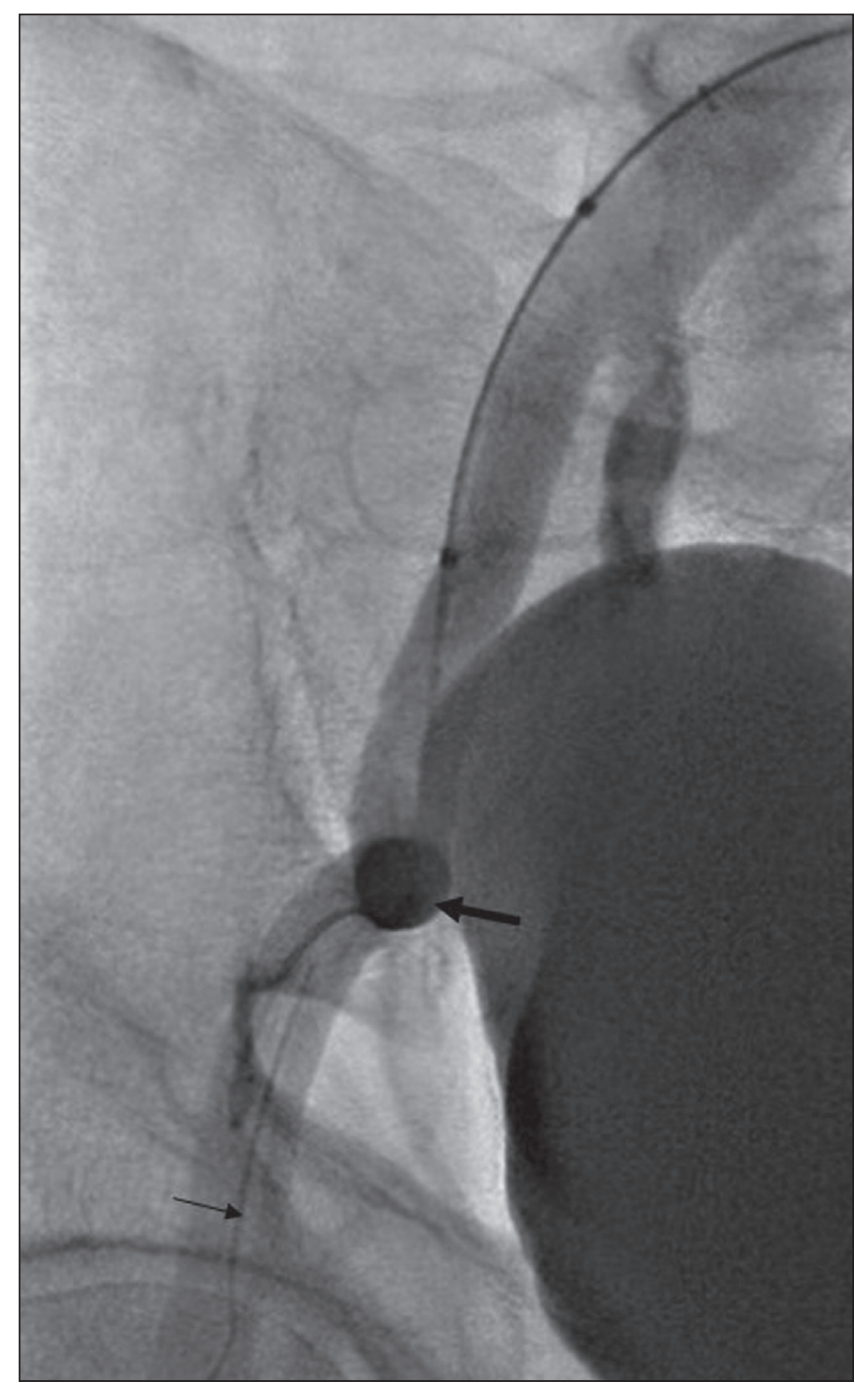

Figure 6) Following partial withdrawal of the super-stiff guidewire, a tortuous loop (large arrow) is apparent in the right external iliac artery. The snapped piece of Terumo guidewire (Terumo Medical Corporation, USA) (small arrow) is also apparent within the right femoral sheath

with contrast injection after partial withdrawal of the Amplatz SupeStiff wire revealed a 'tortuous loop' in the right EIA, exactly at the site where the catheter knot had been entrapped (Figure 6). This 'loop' was not visible on the preceding fluoroscopy view (Figure 3), indicating that the Amplatz Super-Stiff wire had first straightened the tortuous loop in the right EIA, thus aiding in the dislodgement of the impacted catheter knot by the angioplasty balloon. Following removal of the guide catheter remnant, fluoroscopy showed no dissection or pseudoaneurysm within the right CFA or EIA, but the snapped end of the Terumo wire could be seen within the right femoral sheath (Figure 6). The latter was pulled out under fluoroscopic guidance and it was possible to confirm that the wire remnant had safely been retrieved with it. Hemostasis of the left femoral artery was achieved using the Prostar XL vascular closure device (Abbott Vascular, USA), while right femoral artery hemostasis was achieved using manual compression at a later stage. Inspection of both groins the following morning revealed no hematoma or bruising, and an ultrasound scan of both sites showed no vascular complications, including pseudoaneurysm or hematoma. The patient was transferred back to his local district hospital the next day and was discharged home two days later with no reported complications. 


\section{DISCUSSION}

In transfemoral cardiac catheterization, retrieval of a knotted catheter through the ipsilateral femoral artery can be challenging. Simple counter torquing of the catheter may be ineffective at correcting the deformity or, potentially, make it even worse, while aggressive traction may result in vessel trauma, including dissection and perforation $(3,4)$. Often, the kink or knot within the catheter can be straightened using a standard or stiff guide wire, but where this manoeuvre fails, the use of a snaring device may be necessary $(5,6)$. In the present case, we were able to retrieve part of the catheter from the contralateral femoral artery using a GooseNeck snare device but, due to snapping of the catheter, the proximal knotted half remained firmly impacted within the right EIA. An innovative approach of balloon inflation distal to the catheter knot followed by gentle traction on the balloon catheter enabled us to dislodge the knot with subsequent retrieval of the catheter remnant from the contralateral femoral side. The mechanism responsible for the successful dislodgement of the knot using the above technique probably relied on a combination of lumen enlargement with the angioplasty balloon and straightening of the tortuous vessel segment with the super stiff wire. Inflation of the balloon close to the site of impaction 'loosened' the knot. Gentle traction on the inflated balloon subsequently enabled displacement of the knot cephalad into the straightened vessel segment, in a manner analogous to extrusion of toothpaste from its tube. The risk of catheter knotting with a transfemoral approach is greater when the iliofemoral vessels are tortuous and/or calcified, a particularly common situation in elderly patients and individuals with peripheral vascular disease. The use of long sheaths $(23 \mathrm{~cm})$ and long exchange length guide wires $(260 \mathrm{~cm}$ to 300 $\mathrm{cm})$ can mitigate this risk. In the present case, the presence of a tortuous EIA predisposed to this rare complication. Interventional cardiologists and radiologists need to be aware of this important complication and the percutaneous techniques available to safely manage it, thus averting the need for surgical extraction $(7,8)$.
DISCLOSURES: The authors have no financial disclosures or conflicts of interest to declare.

\section{REFERENCES}

1. Tanner MA, Ward D. Percutaneous technique for the reduction of knotted coronary catheters. Heart 2003;89:1132-3.

2. Rafie IM, Viswanathan G, Penny WJ. Transfemoral contralateral technique to retrieve knotted coronary artery catheter using Amplatz Goose Neck snare catheter. BMJ Case Reports 2010;10.1136/bcr.12.2009.2598, 2010 November 19.

3. Younes Nozary, Omid Hashemi Fard. Rare occurrence of simultaneous dissection, perforation and thrombosis of external iliac artery following diagnostic coronary angiography: A case report. ARYA Atheroscler 2010;6:82-4.

4. Prasad A, Compton PA, Prasad A, et al. Incidence and treatment of arterial access dissections occurring during cardiac catheterization. J Interv Cardiol 2008;21:61-6.

5. Rodrigues D, Sá e Melo A, da Silva AM, Carvalheiro V, Manuel O. Percutaneous retrieval of foreign bodies from the cardiovascular system. Rev Port Cardiol 2007;26:755-8.

6. Yedlicka JW Jr, Carlson JE, Hunter DW, Castañeda-Zúñiga WR, Amplatz K. Nitinol gooseneck snare for removal of foreign bodies: Experimental study and clinical evaluation. Radiology 1991;178:691-3.

7. Papakostas JC, Papadopoulos LS, Arnaoutoglou HM, Karahaliou A, Matsagas MI. Transfemoral removal of a knotted Swan-Ganz catheter. Can J Surg 2008;51:E107-8.

8. Alexander LL, Kim SU, Ozoktay S, Horowitz LM. Intra-arterial knotted catheter. J Natl Med Assoc 1978;70:643. 